\title{
Finite-Time Adaptive Synchronization of a New Hyperchaotic System with Uncertain Parameters
}

\author{
Ma Yongguang and Dong Zijian \\ School of Control and Computer Engineering, North China Electric Power University, Baoding 071003, China \\ Correspondence should be addressed to Ma Yongguang; yongguang_ma@163.com
}

Received 25 June 2014; Revised 11 September 2014; Accepted 11 September 2014; Published 13 October 2014

Academic Editor: Baocang Ding

Copyright ( 2014 M. Yongguang and D. Zijian. This is an open access article distributed under the Creative Commons Attribution License, which permits unrestricted use, distribution, and reproduction in any medium, provided the original work is properly cited.

This paper presents a finite-time adaptive synchronization strategy for a class of new hyperchaotic systems with unknown slave system's parameters. Based on the finite-time stability theory, an adaptive control law is derived to make the states of the new hyperchaotic systems synchronized in finite-time. Numerical simulations are presented to show the effectiveness of the proposed finite time synchronization scheme.

\section{Introduction}

Chaos synchronization has attracted increasing attention since the pioneering work of Pecora and Carroll [1] for its potential applications in secure communications, biological systems, chemical reactions, biological networks, and so on. Different notations of synchronization have been proposed, such as complete synchronization [2], generalized synchronization $[3,4]$, phase synchronization $[5,6]$, lag synchronization $[7,8]$, antisynchronization $[9,10]$, and projective synchronization [11, 12]. The idea of synchronization is to use the output of the master system to control the slave system so that the output of the slave system follows the output of the master system asymptotically. A wide variety of synchronization approaches have been developed such as impulsive control [13], feedback control [14], active control [15], adaptive control [16, 17], sliding mode control [18], model predictive control [19-22], and impulsive control [23, $24]$ and others $[25,26]$. In the last thirty years, as hyperchaos has more than one positive Lyapunov exponent and has more complex dynamical behavior than chaos, many researches have focused their attention on the synchronization of hyperchaotic systems [27-31].

However, in real-world application, it is usually expected that two systems can synchronize as quickly as possible and the finite-time control is an efficient technique [3237]. Furthermore, the finite-time control techniques have demonstrated better robustness and disturbance rejection properties [38]. However, most of the results are derived based on the hypothesis that the system's parameters are precisely known. But in practice, most of the system's parameters cannot be exactly known in advance. The designed synchronization will be destroyed with the effects of these uncertainties. To the best of our knowledge, there is no work on the problem of finite-time adaptive synchronization of hyperchaotic systems with uncertain parameters. Motivated by the above discussions, in this paper, we are concerned with the finite-time adaptive synchronization for hyperchaotic systems. Via adaptive control method, finite-time adaptive synchronization between two identical hyperchaotic systems with unknown parameters is achieved and we prove that the suggested approach can realize finite-time synchronization. Simulation results show the effectiveness of the proposed method.

\section{The Dadras System and Lemmas}

Recently, a new 4D dynamical system is proposed [39], which can generate a four-ring hyperchaotic attractor and a fourwing chaotic attractor. The system is called Dadras system in this paper and it is described by

$$
\begin{gathered}
\dot{x}=a x-y z+w \\
\dot{y}=x z-b y
\end{gathered}
$$



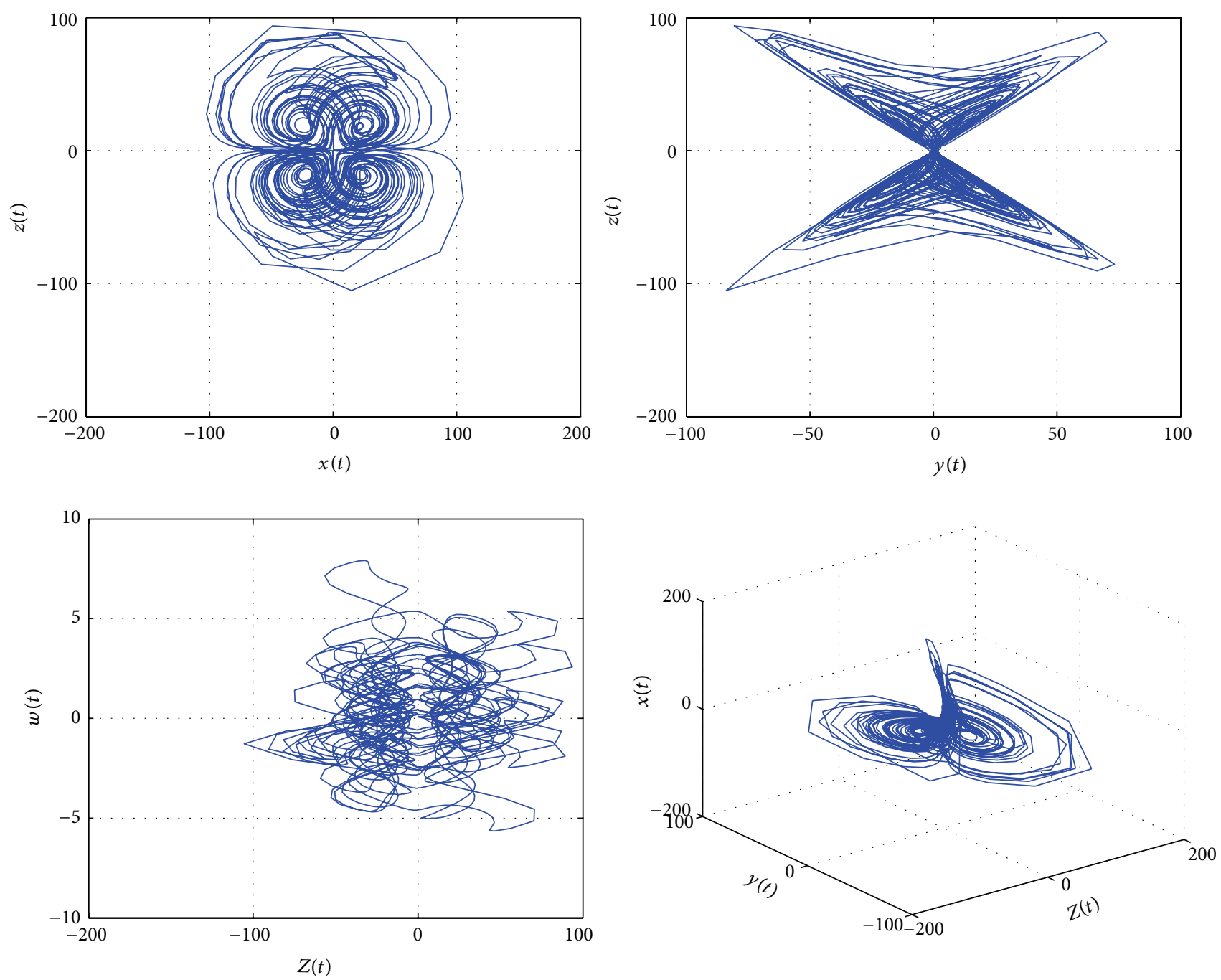

FIgURE 1: Phase portraits of the four-wing hyperchaotic attractor for $a=8, b=40$, and $c=14.9$.

$$
\begin{gathered}
\dot{z}=x y-c z+x w \\
\dot{w}=-y,
\end{gathered}
$$

where $[x, y, z, w]^{T} \in R^{4}$ is the state vector and $a, b$, and $c$ are positive constant parameters of the system. When $a=8, b=$ $40, c=14.9$, and the initial condition is set to $[10,1,10,1]^{T}$, the system has generated a four-wing hyperchaotic attractor which is shown in Figure 1.

Lemma 1 (see [40]). Assume that a continuous, positivedefinite function $V(t)$ satisfies the following differential inequality:

$$
\dot{V}(t) \leq-\gamma V^{\eta}(t) \quad \forall t \geq t_{0}, V\left(t_{0}\right) \geq 0,
$$

where $\gamma>0,0<\eta<1$, are all constants. Then, for any given $t_{0}, V(t)$ satisfies the following inequality:

$$
\begin{gathered}
V^{1-\eta}(t) \leq V^{1-\eta}\left(t_{0}\right)-\gamma(1-\eta)\left(t-t_{0}\right), \quad t_{0} \leq t \leq t_{1} \\
V(t) \equiv 0 \quad \forall t \geq t_{1}
\end{gathered}
$$

with $t_{1}$ given by

$$
t_{1}=t_{0}+\frac{V^{1-\eta}\left(t_{0}\right)}{\gamma(1-\eta)}
$$

\section{The Proposed Synchronization Method}

In order to achieve master-slave synchronization of Dadras system, the master and slave systems are defined with the subscripts $m$ and $s$ below, respectively:

$$
\begin{gathered}
\dot{x}_{m}=a x_{m}-y_{m} z_{m}+w_{m} \\
\dot{y}_{m}=x_{m} z_{m}-b y_{m} \\
\dot{z}_{m}=x_{m} y_{m}-c z_{m}+x_{m} w_{m} \\
\dot{w}_{m}=-y_{m},
\end{gathered}
$$



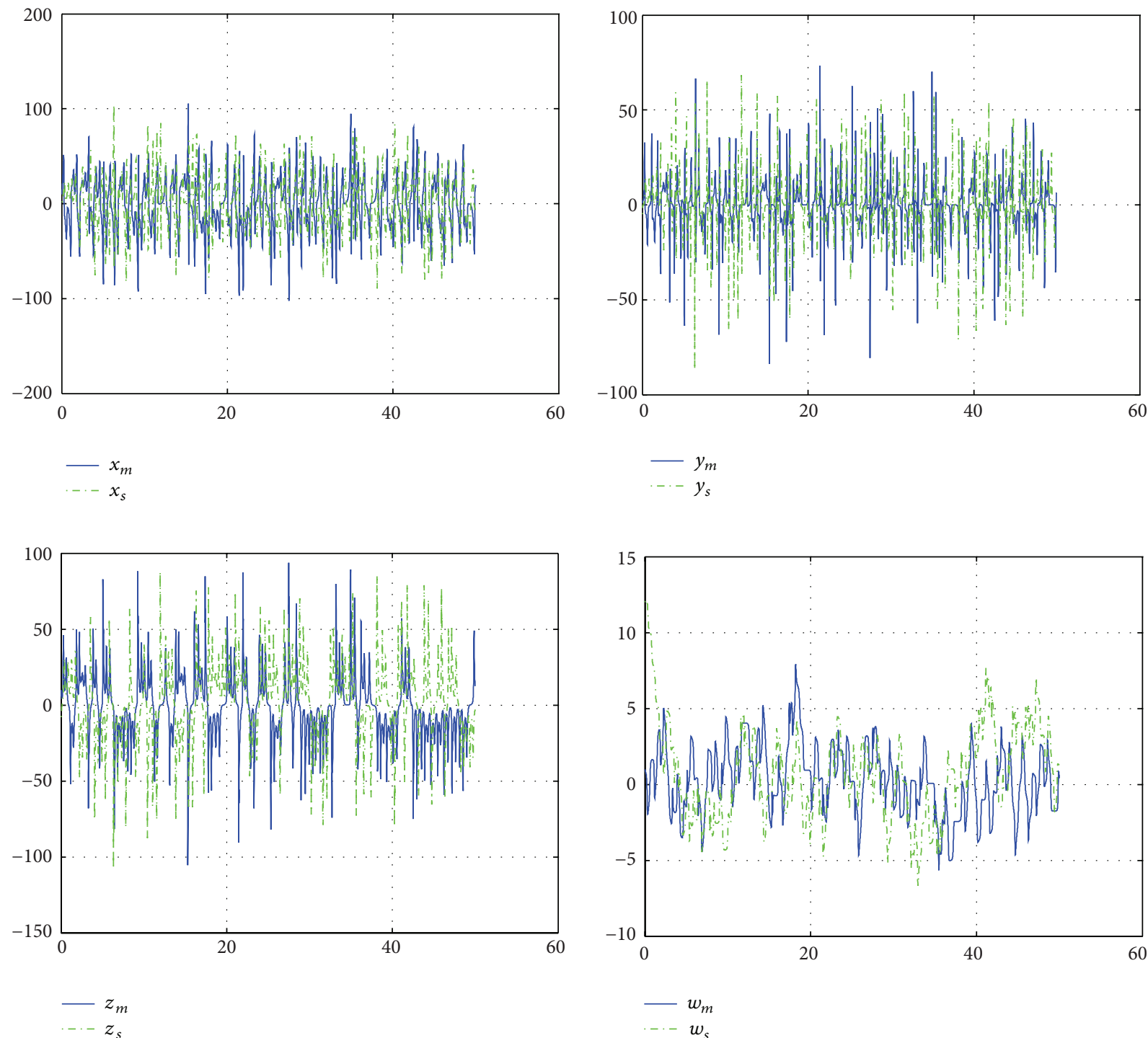

FIGURE 2: States of the master and slave systems without control.

$$
\begin{gathered}
\dot{x}_{s}=a_{s} x_{s}-y_{s} z_{s}+w_{s}+u_{1} \\
\dot{y}_{s}=x_{s} z_{s}-b_{s} y_{s}+u_{2} \\
\dot{z}_{s}=x_{s} y_{s}-c_{s} z_{s}+x_{s} w_{s}+u_{3} \\
\dot{w}_{s}=-y_{s}+u_{4},
\end{gathered}
$$

where $a_{s}, b_{s}$, and $c_{s}$ are uncertain parameters, which need to be estimated in the slave system, and $u_{1}, u_{2}, u_{3}$, and $u_{4}$ are the designed controllers to realize the two hyperchaotic systems' finite-time synchronization.

Let

$$
\begin{gathered}
e_{1}=x_{s}(t)-x_{m}(t) \\
e_{2}=y_{s}(t)-y_{m}(t) \\
e_{3}=z_{s}(t)-z_{m}(t) \\
e_{4}=w_{s}(t)-w_{m}(t) .
\end{gathered}
$$

Then the error dynamical system between (2) and (3) is

$$
\begin{gathered}
\dot{e}_{1}=a_{s} x_{s}-y_{s} z_{s}+w_{s}-a x_{m}+y_{m} z_{m}-w_{m}+u_{1} \\
\dot{e}_{2}=x_{s} z_{s}-b_{s} y_{s}-x_{m} z_{m}+b y_{m}+u_{2} \\
\dot{e}_{3}=x_{s} y_{s}-c_{s} z_{s}+x_{s} w_{s}-x_{m} y_{m}+c z_{m}-x_{m} w_{m}+u_{3} \\
\dot{e}_{4}=-y_{s}+y_{m}+u_{4} .
\end{gathered}
$$

Our goal is to design controllers $u_{i}(i=1,2,3,4)$ to realize finite-time synchronization between the master system (6) and the slave system (7) with the uncertain slave system parameters; that is, $\|e(t)\|=0$ when $t>T_{0}$, where $e=\left[e_{1}, e_{2}, e_{3}, e_{4}\right]^{T}$. 

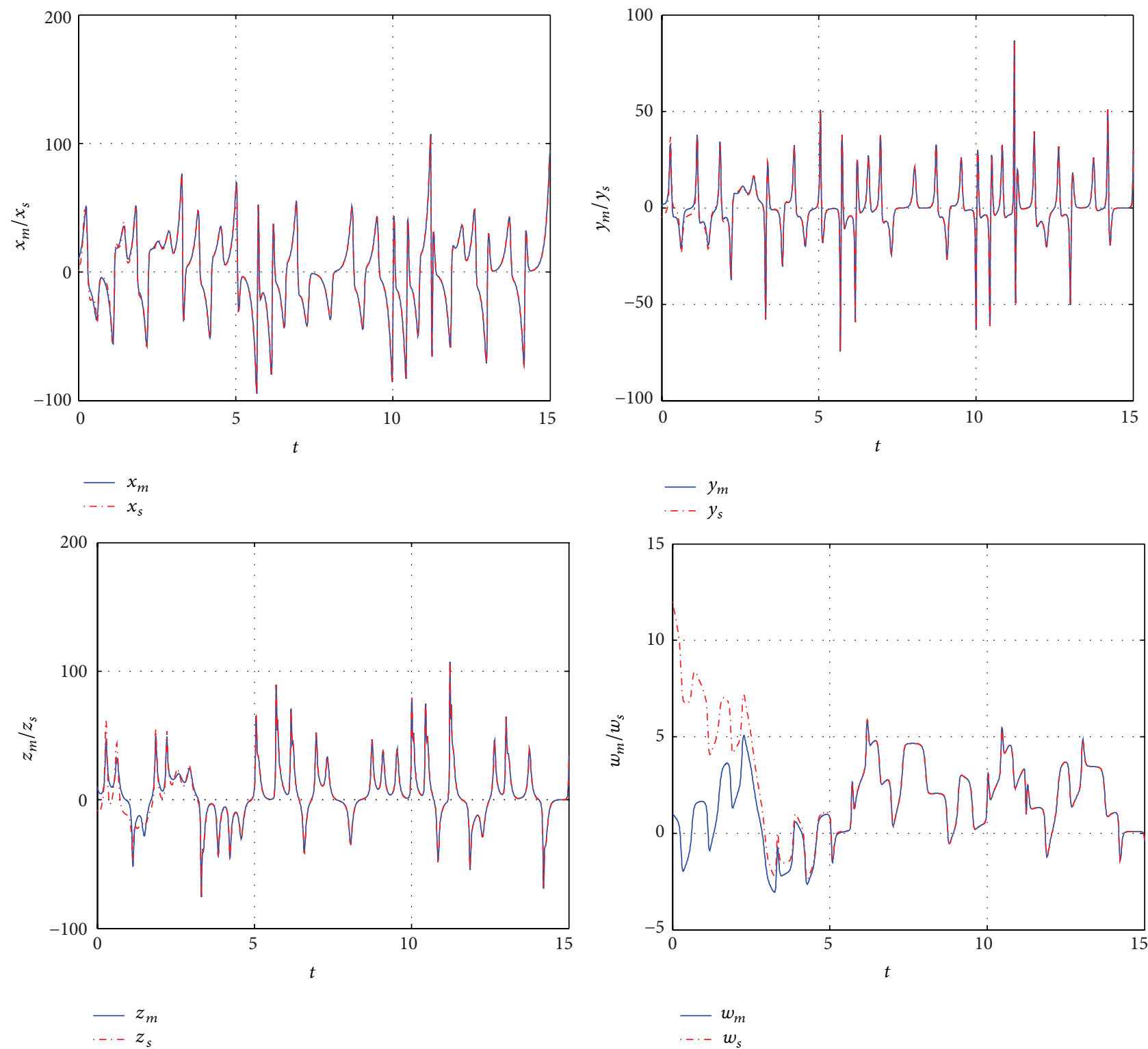

FIGURE 3: States of the master and slave systems with the designed controller.

Define

$$
\begin{aligned}
& e_{a}=a_{s}-a \\
& e_{b}=b_{s}-b \\
& e_{c}=c_{s}-c .
\end{aligned}
$$

Then (5) can be converted to the following form:

$$
\begin{gathered}
\dot{e}_{1}=e_{a} x_{s}+a e_{1}-y_{m} e_{3}-z_{s} e_{2}+e_{4}+u_{1} \\
\dot{e}_{2}=x_{s} e_{3}+z_{m} e_{1}-b_{s} e_{2}-y_{m} e_{b}+u_{2} \\
\dot{e}_{3}=x_{s} e_{2}+y_{m} e_{1}-c_{s} e_{3}-z_{m} e_{c}+x_{s} e_{4}+w_{m} e_{1}+u_{3} \\
\dot{e}_{4}=-e_{2}+u_{4} .
\end{gathered}
$$

In order to achieve the synchronization, we select the following control laws and the update rules for three uncertain parameters $a_{s}, b_{s}$, and $c_{s}$ :

$$
\begin{gathered}
u_{1}=-a e_{1}+y_{m} e_{3}+z_{s} e_{2}-e_{4}-e_{1}^{\beta} \\
u_{2}=-x_{s} e_{3}-z_{m} e_{1}+b_{s} e_{2}-e_{2}^{\beta} \\
u_{3}=-x_{s} e_{2}-y_{m} e_{1}+c_{s} e_{3}-x_{s} e_{4}-w_{m} e_{1}-e_{3}^{\beta} \\
u_{4}=e_{2}-e_{4}^{\beta}, \\
\dot{e}_{a}=-e_{1} x_{s}-e_{a}^{\beta} \\
\dot{e}_{b}=y_{m} e_{2}-e_{b}^{\beta} \\
\dot{e}_{c}=z_{m} e_{3}-e_{c}^{\beta} .
\end{gathered}
$$

Then, the following result is obtained. 
Mathematical Problems in Engineering

5
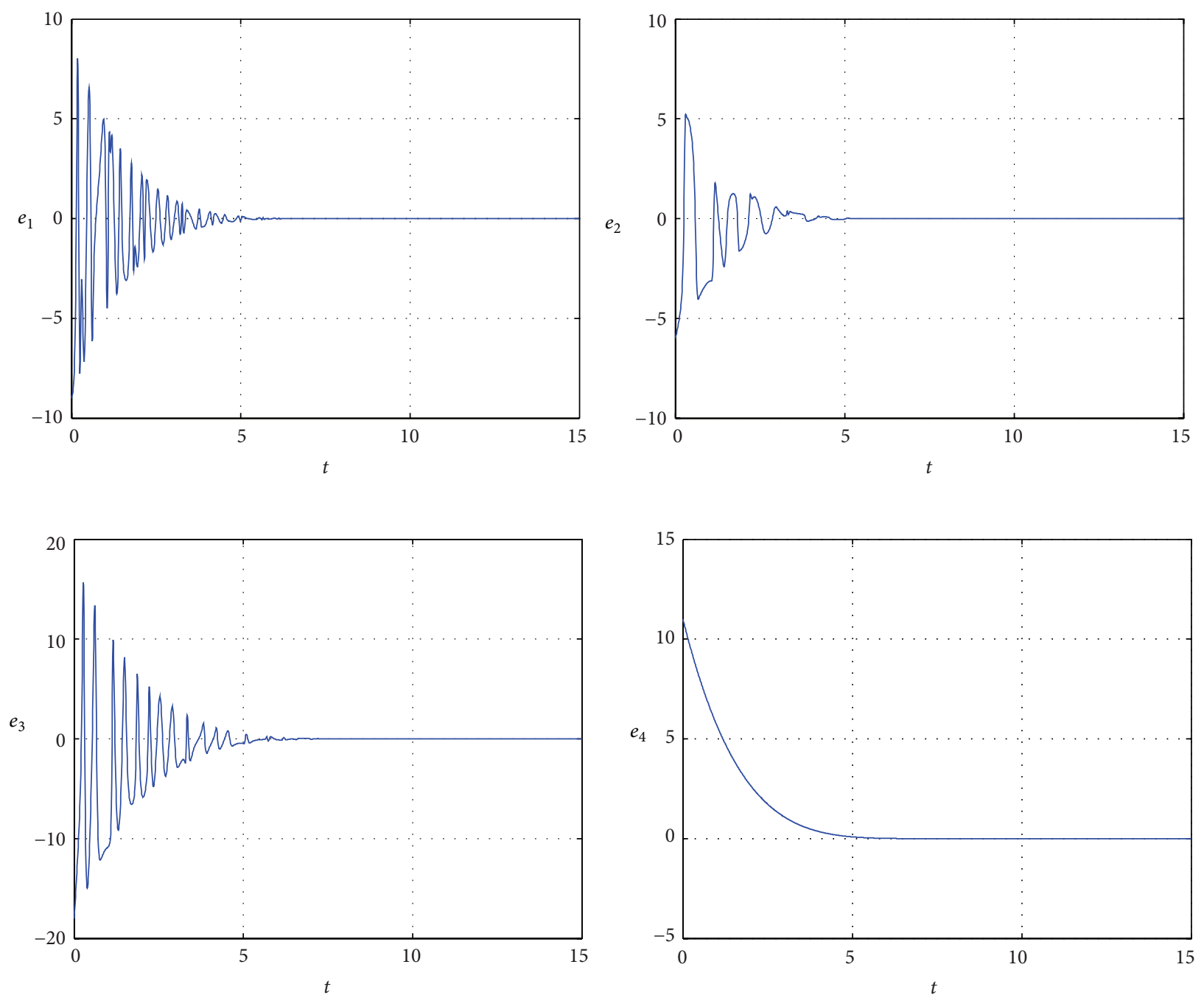

FIGURE 4: Synchronization errors between the master and slave systems with the designed controller.

Theorem 2. For any initials, the two systems (6) and (7) realize finite-time adaptive synchronization under the control laws (12) and the parameters' update laws (13).

Proof. Choose the following Lyapunov function candidate:

$$
V=\frac{1}{2}\left(e_{1}^{2}+e_{2}^{2}+e_{3}^{2}+e_{4}^{2}+e_{a}^{2}+e_{b}^{2}+e_{c}^{2}\right) .
$$

The differential of the Lyapunov function along the trajectory of the error system (13) is

$$
\begin{aligned}
\frac{d V}{d t}= & e_{1}\left(e_{a} x_{s}-e_{1}^{\beta}\right)+e_{2}\left(-y_{m} e_{b}-e_{2}^{\beta}\right)+e_{3}\left(-z_{m} e_{c}-e_{3}^{\beta}\right) \\
& +e_{4}\left(-e_{4}^{\beta}\right)+e_{a}\left(-e_{1} x_{s}-e_{a}^{\beta}\right)+e_{b}\left(y_{m} e_{2}-e_{b}^{\beta}\right) \\
& +e_{c}\left(z_{m} e_{3}-e_{c}^{\beta}\right) \\
= & -e_{1}^{1+\beta}-e_{2}^{1+\beta}-e_{3}^{1+\beta}-e_{4}^{1+\beta}-e_{a}^{1+\beta}-e_{b}^{1+\beta}-e_{c}^{1+\beta}
\end{aligned}
$$

$$
\begin{aligned}
=-2^{(\beta+1) / 2}( & \left(\frac{1}{2} e_{1}^{2}\right)^{(\beta+1) / 2}+\left(\frac{1}{2} e_{2}^{2}\right)^{(\beta+1) / 2} \\
& +\left(\frac{1}{2} e_{3}^{2}\right)^{(\beta+1) / 2}+\left(\frac{1}{2} e_{4}^{2}\right)^{(\beta+1) / 2} \\
& +\left(\frac{1}{2} e_{a}^{2}\right)^{(\beta+1) / 2}+\left(\frac{1}{2} e_{b}^{2}\right)^{(\beta+1) / 2} \\
& \left.+\left(\frac{1}{2} e_{c}^{2}\right)^{(\beta+1) / 2}\right) \\
\leq-2^{(\beta+1) / 2}( & \frac{1}{2} e_{1}^{2}+\frac{1}{2} e_{2}^{2}+\frac{1}{2} e_{3}^{2}+\frac{1}{2} e_{4}^{2}+\frac{1}{2} e_{a}^{2}+\frac{1}{2} e_{b}^{2} \\
& \left.+\frac{1}{2} e_{c}^{2}\right)^{(\beta+1) / 2} \\
=-2^{(\beta+1) / 2} V^{(\beta+1) / 2} &
\end{aligned}
$$



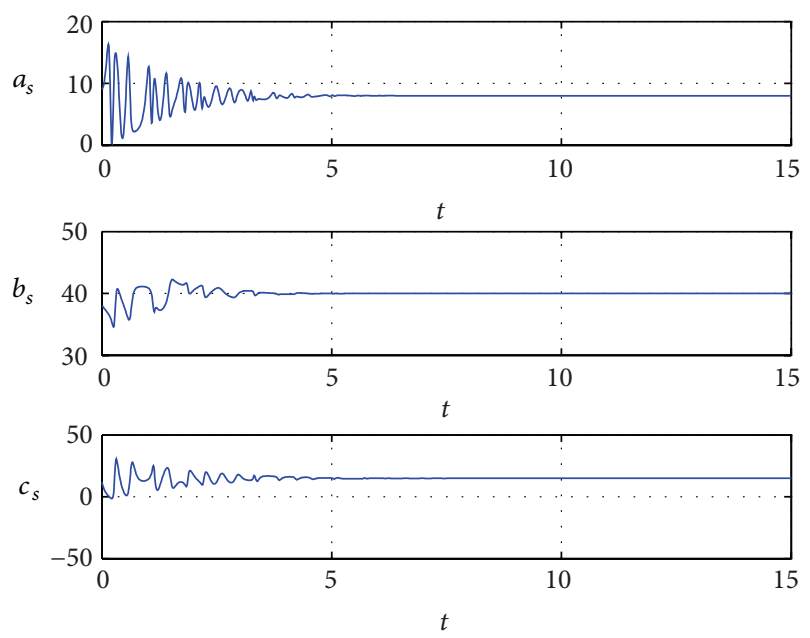

FIGURE 5: Adaptive parameters $a_{s}, b_{s}$, and $c_{s}$.

From Lemma 1, it follows that the error system (11) is finite-time stabilized. Then the uncertain slave system (7) can synchronize the master system (6) in finite time.

Remark 3. From the proof of Theorem 2, it is found that $d V / d t \leq-2^{(\beta+1) / 2} V^{(\beta+1) / 2}$. Using Lemma 1, we can get $V(t) \equiv$ $0, \forall t \geq t_{1}$, where $t_{1}=2^{(1-\beta) / 2} \cdot(1 /(1-\beta))[V(0)]^{(1-\beta) / 2}$. So the synchronization time is influenced by not only the parameters and initial values' mismatch but also the control parameter $\beta$.

Remark 4. Although the synchronization scheme is designed for Dadras systems, it can be used in two other identical hyperchaotic systems and even two different hyperchaotic systems.

\section{Simulation Results}

Numerical simulation results are presented to show the effectiveness of the proposed finite-time synchronization method. Fourth-order Runge-Kutta method is used and the time step size is $0.001 \mathrm{~s}$. The master system's parameters and initial conditions are the same as in Figure 1. The initial states of the response system are $x_{s}(0)=1, y_{s}(0)=-5$, $z_{s}(0)=-8$, and $w_{s}(0)=12$. Furthermore, the initial values of estimated parameters are $a=9, b=38$, and $c=12$ and the parameter $\beta=0.8$. The simulations of the two Dadras systems without control are shown in Figure 2, followed by the simulation with the designed finite-time adaptive control shown in Figure 3. Figure 4 shows that the trajectories of $e_{1}(t), e_{2}(t), e_{3}(t)$, and $e_{4}(t)$ tended to zero in finite time. The changes of parameters of $a_{s}, b_{s}$, and $c_{s}$ are shown in Figure 5. Obviously, the synchronization errors converge to zero and the estimations of parameters converge to some constants in finite time.

\section{Conclusion}

This paper has addressed the finite-time adaptive synchronization of Dadras hyperchaotic systems. Based on finitetime stability theory, the proposed scheme can assure the states of slave system to track the states of the master system in finite time. From the process of proof we can see that this method can be extended to other hyperchaotic systems such as Rössler hyperchaotic system and Lü hyperchaotic system.

\section{Conflict of Interests}

The authors declare that there is no conflict of interests regarding the publication of this paper.

\section{References}

[1] L. M. Pecora and T. L. Carroll, "Synchronization in chaotic systems," Physical Review Letters, vol. 64, no. 8, pp. 821-824, 1990.

[2] C. Li and X. Liao, "Complete and lag synchronization of hyperchaotic systems using small impulses," Chaos, Solitons and Fractals, vol. 22, no. 4, pp. 857-867, 2004.

[3] N. F. Rulkov, M. M. Sushchik, L. S. Tsimring, and H. D. I. Abarbanel, "Generalized synchronization of chaos in directionally coupled chaotic systems," Physical Review E, vol. 51, no. 2, pp. 980-994, 1995.

[4] S. S. Yang and C. K. Duan, "Generalized synchronization in chaotic systems," Chaos, Solitons and Fractals, vol. 9, no. 10, pp. 1703-1707, 1998.

[5] M. G. Rosenblum, A. S. Pikovsky, and J. Kurths, "Phase synchronization of chaotic oscillators," Physical Review Letters, vol. 76, no. 11, pp. 1804-1807, 1996.

[6] G. V. Osipov, A. S. Pikovsky, M. G. Rosenblum, and J. Kurths, "Phase synchronization effects in a lattice of nonidentical Rössler oscillators," Physical Review E, vol. 55, no. 3, pp. 23532361, 1997.

[7] M. G. Rosenblum, A. S. Pikovsky, and J. Kurths, "From phase to lag synchronization in coupled chaotic oscillators," Physical Review Letters, vol. 78, no. 22, pp. 4193-4196, 1997.

[8] Z. Yan, "Q-S (lag or anticipated) synchronization backstepping scheme in a class of continuous-time hyperchaotic systems-a symbolic-numeric computation approach," Chaos: An Interdisciplinary Journal of Nonlinear Science, vol. 15, no. 2, Article ID 023902, 2005.

[9] C.-M. Kim, S. Rim, W.-H. Kye, J.-W. Ryu, and Y.-J. Park, "Antisynchronization of chaotic oscillators", Physics Letters A, vol. 320, no. 1, pp. 39-46, 2003.

[10] Z. Wang, "Anti-synchronization in two non-identical hyperchaotic systems with known or unknown parameters," Communications in Nonlinear Science and Numerical Simulation, vol. 14, no. 5, pp. 2366-2372, 2009.

[11] R. Mainieri and J. Rehacek, "Projective synchronization in three-dimensional chaotic systems," Physical Review Letters, vol. 82, no. 15, pp. 3042-3045, 1999.

[12] D. Xu, Z. Li, and S. R. Bishop, "Manipulating the scaling factor of projective synchronization in three-dimensional chaotic systems," Chaos, vol. 11, no. 3, pp. 439-442, 2001.

[13] Y.-W. Wang, Z.-H. Guan, and J.-W. Xiao, "Impulsive control for synchronization of a class of continuous systems," Chaos, vol. 14, no. 1, pp. 199-203, 2004. 
[14] M. Rafikov and J. M. Balthazar, "On control and synchronization in chaotic and hyperchaotic systems via linear feedback control," Communications in Nonlinear Science and Numerical Simulation, vol. 13, no. 7, pp. 1246-1255, 2008.

[15] H. N. Agiza and M. T. Yassen, "Synchronization of Rossler and Chen chaotic dynamical systems using active control," Physics Letters. A, vol. 278, no. 4, pp. 191-197, 2001.

[16] S. Chen and J. Lü, "Synchronization of an uncertain unified chaotic system via adaptive control," Chaos, Solitons and Fractals, vol. 14, no. 4, pp. 643-647, 2002.

[17] L. Zhang and Y. Yan, "Robust synchronization of two different uncertain fractional-order chaotic systems via adaptive sliding mode control," Nonlinear Dynamics, vol. 76, no. 3, pp. 1761-1767, 2014.

[18] T. Yang and H. H. Shao, "Synchronizing chaotic dynamics with uncertainties based on a sliding mode control design," Physical Review E, vol. 65, no. 4, Article ID 046210, 7 pages, 2002.

[19] L. Zhang and X. Liu, "The synchronization between two discrete-time chaotic systems using active robust model predictive control," Nonlinear Dynamics, vol. 74, no. 4, pp. 905-910, 2013.

[20] B. Ding and B. Huang, "Reformulation of LMI-based stabilisation conditions for non-linear systems in Takagi-Sugeno's form," International Journal of Systems Science, vol. 39, no. 5, pp. 487-496, 2008.

[21] B. Ding, Y. Xi, M. T. Cychowski, and T. O’Mahony, “Improving off-line approach to robust MPC based-on nominal performance cost," Automatica, vol. 43, no. 1, pp. 158-163, 2007.

[22] B. Ding, Y. Xi, M. T. Cychowski, and T. O'Mahony, "A synthesis approach for output feedback robust constrained model predictive control," Automatica, vol. 44, no. 1, pp. 258-264, 2008.

[23] C. Li, G. Chen, X. Liao, and Z. Fan, "Chaos quasisynchronization induced by impulses with parameter mismatches," Chaos: An Interdisciplinary Journal of Nonlinear Science, vol. 16, no. 2, Article ID 023102, 2006.

[24] C. Li, X. Liao, X. Yang, and T. Huang, "Impulsive stabilization and synchronization of a class of chaotic delay systems," Chaos, vol. 15, no. 4, Article ID 043103, 2005.

[25] C. Li and X. Liao, "Lag synchronization of Rossler system and Chua circuit via a scalar signal," Physics Letters A, vol. 329, no. 4-5, pp. 301-308, 2004.

[26] Q. Han, C. Li, and T. Huang, "Anticipating synchronization of a class of chaotic systems," Chaos, vol. 19, no. 2, Article ID 023105, 10 pages, 2009.

[27] Y. Li, W. K. S. Tang, and G. Chen, "Generating hyperchaos via state feedback control," International Journal of Bifurcation and Chaos in Applied Sciences and Engineering, vol. 15, no. 10, pp. 3367-3375, 2005.

[28] D. Cafagna and G. Grassi, "New 3D-scroll attractors in hyperchaotic Chua's circuits forming a ring," International Journal of Bifurcation and Chaos in Applied Sciences and Engineering, vol. 13, no. 10, pp. 2889-2903, 2003.

[29] O. E. Rossler, "An equation for hyperchaos," Physics Letters A, vol. 71, no. 2-3, pp. 155-157, 1979.

[30] F. Wang and C. Liu, "A new criterion for chaos and hyperchaos synchronization using linear feedback control," Physics Letters Section A, vol. 360, no. 2, pp. 274-278, 2006.

[31] N. Yang and C. Liu, "A novel fractional-order hyperchaotic system stabilization via fractional sliding-mode control," Nonlinear Dynamics, vol. 74, no. 3, pp. 721-732, 2013.
[32] Y. Ying and C. Guopei, "Finite time control of a class of timevarying unified chaotic systems," Chaos, vol. 23, no. 3, Article ID 033143, 2013.

[33] M. P. Aghababa, "Finite-time chaos control and synchronization of fractional-order nonautonomous chaotic (hyperchaotic) systems using fractional nonsingular terminal sliding mode technique," Nonlinear Dynamics, vol. 69, no. 1-2, pp. 247-261, 2012.

[34] M. P. Aghababa and H. P. Aghababa, "A general nonlinear adaptive control scheme for finite-time synchronization of chaotic systems with uncertain parameters and nonlinear inputs," Nonlinear Dynamics, vol. 69, no. 4, pp. 1903-1914, 2012.

[35] N. Cai, W. Li, and Y. Jing, "Finite-time generalized synchronization of chaotic systems with different order," Nonlinear Dynamics, vol. 64, no. 4, pp. 385-393, 2011.

[36] Y. Liu, "Circuit implementation and finite-time synchronization of the 4D Rabinovich hyperchaotic system," Nonlinear Dynamics, vol. 67, no. 1, pp. 89-96, 2012.

[37] U. E. Vincent and R. Guo, "Finite-time synchronization for a class of chaotic and hyperchaotic systems via adaptive feedback controller," Physics Letters, Section A: General, Atomic and Solid State Physics, vol. 375, no. 24, pp. 2322-2326, 2011.

[38] W. Yu, "Finite-time stabilization of three-dimensional chaotic systems based on CLF," Physics Letters A, vol. 374, no. 30, pp. 3021-3024, 2010.

[39] S. Dadras, H. R. Momeni, G. Qi, and Z. 1. Wang, "Fourwing hyperchaotic attractor generated from a new 4D system with one equilibrium and its fractional-order form," Nonlinear Dynamics, vol. 67, no. 2, pp. 1161-1173, 2012.

[40] H. Wang, Z.-Z. Han, Q.-Y. Xie, and W. Zhang, "Finite-time chaos synchronization of unified chaotic system with uncertain parameters," Communications in Nonlinear Science and Numerical Simulation, vol. 14, no. 5, pp. 2239-2247, 2009. 


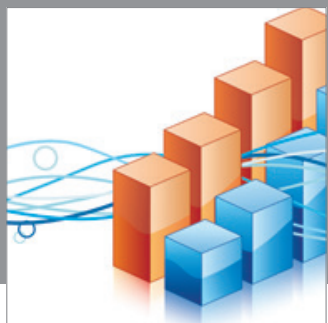

Advances in

Operations Research

mansans

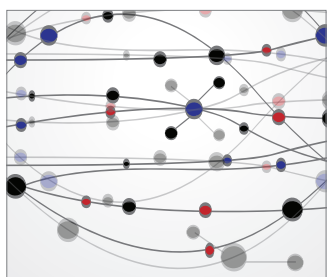

The Scientific World Journal
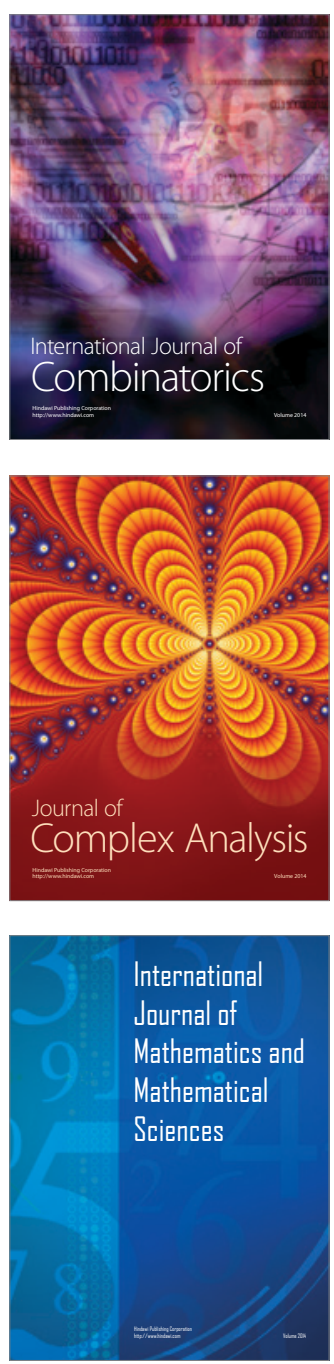
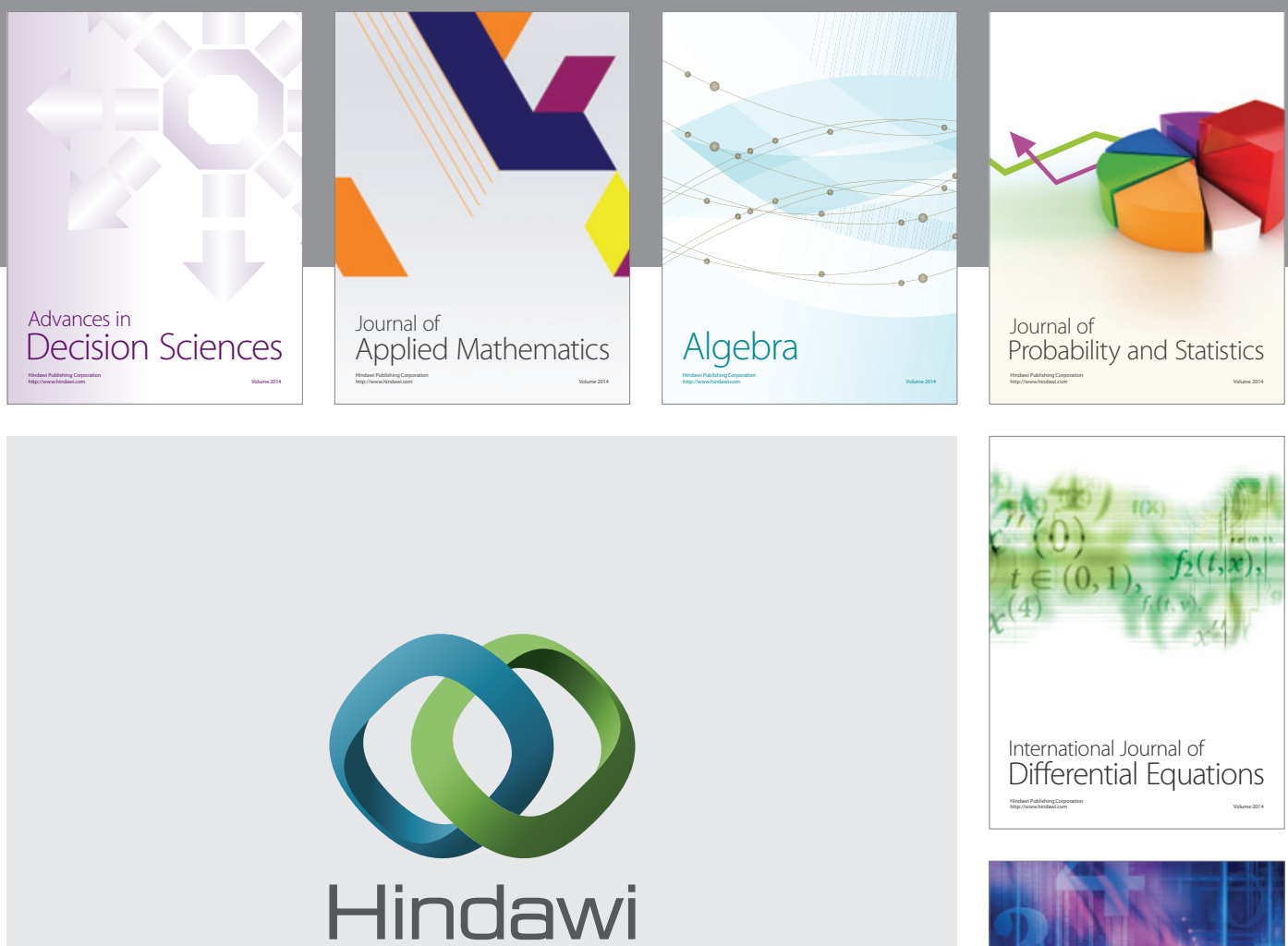

Submit your manuscripts at http://www.hindawi.com
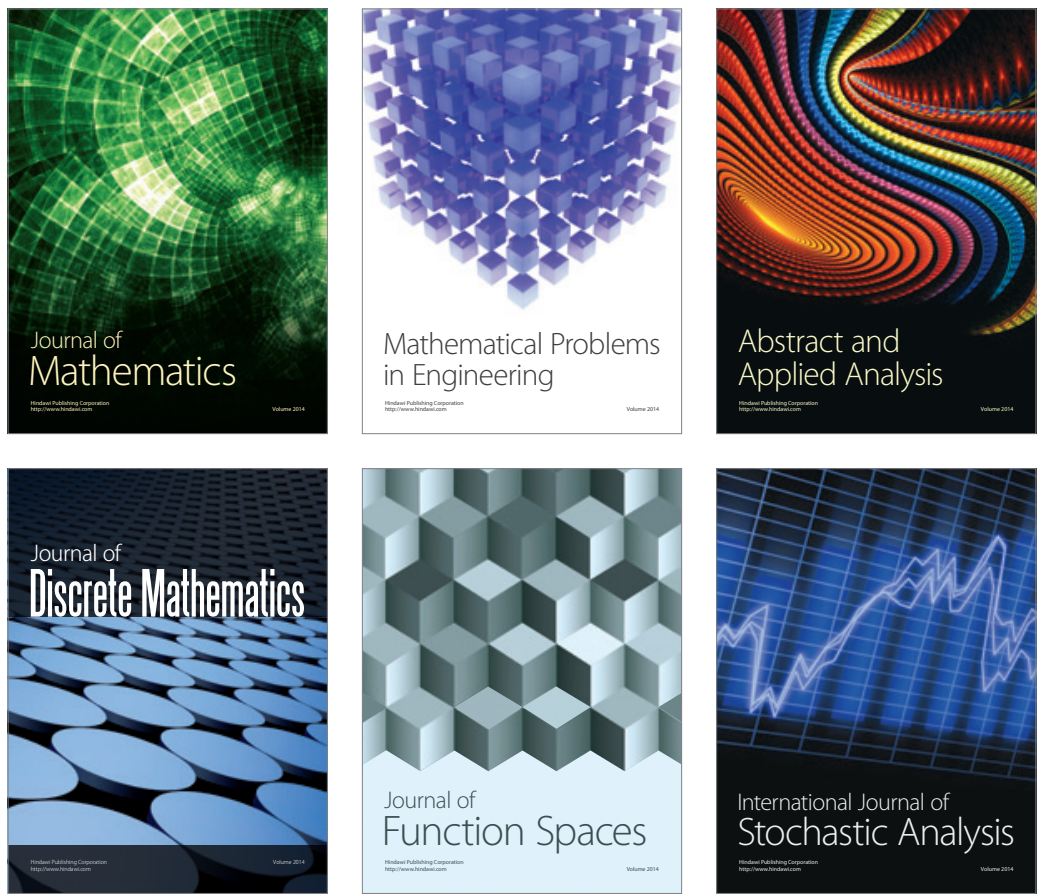

Journal of

Function Spaces

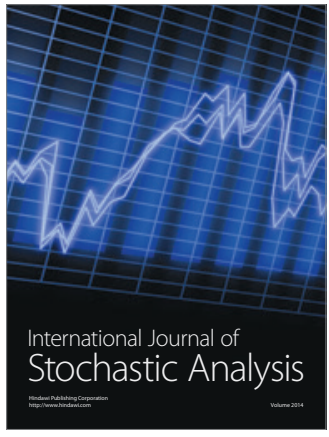

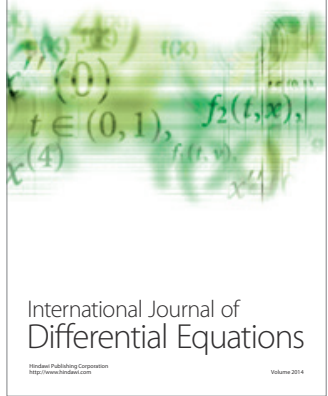
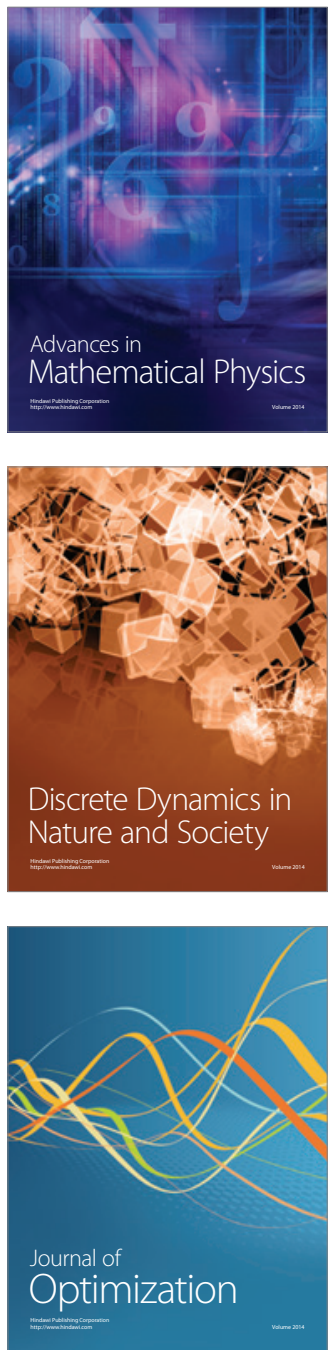